\title{
DECOMPOSABILITY OF RADON MEASURES
}

\author{
BY
}

\author{
R. J. GARDNER AND W. F. PFEFFER
}

\begin{abstract}
A topological space is called metacompact or metalindelöf if each open cover has a point-finite or point-countable refinement, respectively. It is well known that each Radon measure is expressible as a sum of Radon measures supported on a disjoint family of compact sets, called a concassage. If the unions of measurable subsets of the members of a concassage are also measurable, the Radon measure is called decomposable. We show that Radon measures in a metacompact space are always saturated, and therefore decomposable whenever they are complete. The previous statement is undecidable in ZFC if "metacompact" is replaced by "metalindelöf". The proofs are based on structure theorems for a concassage of a Radon measure. These theorems also show that the union of a concassage of a Radon measure in a metacompact space is a Borel set, which is paracompact in the subspace topology whenever the main space is regular.
\end{abstract}

1. Introduction. Two classes of measures have particular importance in measure theory: Maharam or "localizable" measures (in connection with the duality between $L^{1}$ and $L^{\infty}$-see $\left[\mathbf{F}_{1}, 53 \mathrm{~B}\right]$ ), and decomposable or "strictly localizable" measures (in connection with liftings for $L^{\infty}$ - see [II, Chapter IV, §3]). Consequently, they have been extensively studied in both abstract and topological settings.

A decomposable measure is always Maharam, but the converse is false in general (see $\left[\mathbf{F}_{3}, 5\right]$ ). Complete and saturated Radon measures are decomposable, but if either completeness or saturatedness is missing, Radon measures need not be Maharam. The two examples which show this are due to D. H. Fremlin (see $\left.\left[\mathbf{F}_{2}\right]\right)$; in essence, they are obtained by ingenious modifications of the product topology in $\omega_{1} \times[0,1]^{\omega_{1}}$.

Work during the last two decades in topological measure theory has shown that measures in a space which is paracompact or has some weaker covering property (such as metacompactness or weak $\theta$-refinability) are substantially better behaved than those in general spaces; see $\left[\mathbf{G P}_{\mathbf{3}}\right]$ for a self-contained exposition. In this paper, we apply these techniques to the problem of when a Radon measure is decomposable.

By obtaining structure theorems for the concassage of a Radon measure in spaces with certain weak covering properties, we are able to prove, for example, that a Radon measure in a weakly $\theta$-refinable space is always saturated, and hence decomposable whenever it is complete. This shows that the topological complexity of

Received by the editors March 1, 1983 and, in revised form, May 23, 1983.

1980 Mathematics Subject Classification. Primary 28C15, 54D20; Secondary 54A35.

Key words and phrases. Radon measures, Maharam measures, decomposable measures, metacompact and metalindelöf spaces, weakly $\theta$-refinable spaces, continuum hypothesis, Martin's axiom. 
Fremlin's examples is unavoidable. When we replace "weakly $\theta$-refinable" by "metalindelöf", the decomposability of a complete Radon measure becomes undecidable within the usual axioms of set theory; a phenomenon which has been noted before in different situations (see, e.g., $\left[\mathbf{G P}_{\mathbf{3}}\right]$ ). The results concerning concassages may also be of interest in their own right; for example, the union of a concassage of a Radon measure in a metacompact space $X$ is a Borel set, which is paracompact in the subspace topology whenever $X$ is regular.

2. Preliminaries. If $A$ is a set, we denote by $|A|$ its cardinality. Throughout, we shall identify an ordinal with the set of all smaller ordinals. As usual, $\omega$ and $\omega_{1}$ stand for the first infinite and first uncountable ordinals, respectively. The continuum hypothesis and Martin's axiom are abbreviated as $\mathrm{CH}$ and MA, respectively.

If $(X, \mathfrak{N}, \mu)$ is a measure space, we set

$$
\mathfrak{M}_{f}=\{A \in \mathfrak{M}: \mu(A)<+\infty\}, \quad \mathfrak{M}_{0}=\{A \in \mathfrak{M}: \mu(A)=0\} .
$$

Let $(X, \mathfrak{N}, \mu$ ) be a measure space. The measure $\mu$ is called

(i) complete if $A \in \Re$ whenever $A \subset B$ for some $B \in \Re_{0}$;

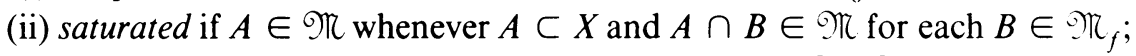

(iii) Maharam if each subset of the measure algebra $\mathscr{N} / \mathcal{O} \mathbb{R}_{0}$ has a least upper bound.

A decomposition of the measure $\mu$ is a disjoint family $\mathcal{H} \subset \mathscr{M}_{f}$ such that

(a) $A \in \mathfrak{N}$ whenever $A \subset X$ and $A \cap H \in \mathfrak{N}$ for each $H \in \mathscr{K}$;

(b) if $A \in \mathscr{N}$, then

$$
\mu(A)=\sum_{H \in \mathcal{K}} \mu(A \cap H) .
$$

If a decomposition of $\mu$ exists, the measure $\mu$ is called decomposable.

If $\mathcal{Q}$ is a family of subsets of a set $E$, and $x \in E$, we let

$$
\operatorname{st}(x, \mathbb{Q})=\{A \in \mathbb{Q}: x \in A\} \text {. }
$$

We say that $Q$ is, respectively, point-finite or point-countable, if $|s t(x, \mathbb{A})|<\omega$ or $|\operatorname{st}(x, \mathbb{Q})| \leqslant \omega$ for each $x \in E$.

Throughout, all spaces will be Hausdorff. A space $X$ is called, respectively, metacompact or metalindelöf if each open cover has a point-finite or point-countable open refinement. If each open cover of $X$ has an open refinement $\cup_{n \in \omega}{ }{ }_{\mathrm{d}_{n}}$ such that given $x \in X$ there is an $n_{x} \in \omega$ with $1 \leqslant\left|s t\left(x, \mathscr{Q}_{n_{X}}\right)\right|<\omega$, then $X$ is called weakly $\theta$-refinable; if, in addition, each $\mathcal{Q}_{n}$ covers $X$, then $X$ is called $\theta$-refinable. Finally, $X$ satisfies the countable chain condition (abbreviated as ccc) if each disjoint family of open subsets of $X$ is countable.

Let $X$ be a space. By $\mathcal{G}$ and $\mathcal{K}$ we denote the families of all open and compact subsets of $X$, respectively. The Borel $\sigma$-algebra in $X$, i.e., the smallest $\sigma$-algebra in $X$ containing $\mathcal{G}$, is denoted by $\mathscr{B}$. The elements of $\mathscr{B}$ are called Borel sets.

Let $\mathscr{N}$ be a $\sigma$-algebra in $X$. A measure $\mu$ on $\mathscr{N}$ is called a Radon measure in $X$ if the following conditions are satisfied:

(a) $\mathcal{S} \subset \mathfrak{N}$;

(b) each $x \in X$ has a neighborhood $U \in \mathscr{T}_{f}$; 
(c) for each $A \in \mathscr{N}, \mu(A)=\sup \{\mu(K): K \in \mathscr{K}, K \subset A\}$.

A concassage of a Radon measure $\mu$ in $X$ is a disjoint family $\mathscr{D}$ of nonempty compact sets such that

(a) if $D \in \mathscr{Q}, G \in \mathcal{G}$, and $D \cap G \neq \varnothing$, then $\mu(D \cap G)>0$;

(b) if $A \in \mathfrak{M}$, then

$$
\mu(A)=\sum_{D \in \mathcal{Q}} \mu(A \cap D) .
$$

We recall three known facts.

(A) Each Radon measure has a concassage (see, e.g., [ $\left.\mathbf{G P}_{\mathbf{2}}, 3.4\right]$ ).

(B) Each complete and saturated Radon measure is decomposable (see $\left[\mathbf{F}_{\mathbf{1}}, 72 \mathrm{~B}\right.$ and $73 \mathrm{~B}]$ ).

(C) Each decomposable measure is Maharam (see $\left.\left[\mathrm{F}_{1}, 64 \mathrm{H}\right]\right)$.

In $\left[\mathbf{F}_{1}\right]$, the proof of $(B)$ is given in a roundabout manner via quasi-Radon measures. Thus it appears beneficial to include a short direct proof.

Proof of (B). Let $\mu$ on $\Re$ be a complete and saturated Radon measure in $X$ with a concassage $\mathscr{Q}$. We shall show that $\mathscr{Q}$ is a decomposition of $\mu$. To this end, choose an $A \subset X$ such that $A \cap D \in \Re$ for each $D \in \mathscr{Q}$. If $B \in \Re_{f}$, find a $\sigma$-compact $C \subset B$ with $\mu(B-C)=0$. It follows from the $\sigma$-compactness of $C$ that $\mathscr{Q} *=\{D \in$ श): $C \cap D \neq \varnothing\}$ is countable. Hence

$$
A \cap C=[A \cap(C-\cup \mathscr{D} *)] \cup \underset{D \in \mathscr{Q}^{*}}{\bigcup}[(A \cap D) \cap C]
$$

belongs to $\Re$; for $\mu$ is complete and $\mu\left(C-\cup \mathscr{D}^{*}\right)=0$. Using the completeness of $\mu$ again, we see that $A \cap(B-C) \in \Re$, and consequently $A \cap B \in \Re$. As $\mu$ is saturated, $A \in \mathscr{N}$.

Suppose that $\mu$ on $\mathscr{T}$ is a complete Radon measure in $X$, but that we have no a priori knowledge whether $\mu$ is saturated. Then the previous proof suggests that the decomposability of $\mu$ depends on how well we can disentangle a concassage of $\mu$; and this is the main idea behind the proof of Theorem 3.4.

3. Main results. We begin with a lemma due to F. D. Tall (see [T, 3.7]).

3.1. Lemma. (MA $+\neg \mathrm{CH})$ Let $X$ be a compact space satisfying the ccc. Then each point-countable family of open subsets of $X$ is countable.

Proof. Let $Q=\left\{G_{\alpha}: \alpha \in \omega_{1}\right\}$ be a family of distinct open subsets of $X$, and for each $\alpha \in \omega_{1}$, let $H_{\alpha}=\bigcap_{\beta \in \omega_{1}-\alpha} G_{\beta}$. Using the ccc, it is easy to see that there is a $\gamma \in \omega_{1}$ such that $\mathrm{cl} H_{\alpha}=\mathrm{cl} H_{\gamma}$ for each $\alpha \in \omega_{1}-\gamma$. Now it follows from MA + $\neg \mathrm{CH}$ that the set $D=\bigcap_{\alpha \in \omega_{1}-\gamma} H_{\alpha}$ is nonempty. Clearly, $|\operatorname{st}(x, \mathbb{Q})|=\omega_{1}$ for each $x \in D$.

3.2. Proposition. Let $\mu$ be a Radon measure in $X$ with a concassage $\mathcal{D}$, and let $Y=\cup \mathscr{D}$. Suppose that either of the following conditions is fulfilled:

(a) $X$ is metacompact;

(b) $X$ is metalindelöf and $\mathrm{MA}+\neg \mathrm{CH}$ holds. 
Then we can find a concassage $\mathcal{E}=\cup_{t \in T} \mathcal{E}_{t}$ of $\mu$ having the following properties:

(i) Each member of $\mathcal{E}$ is a clopen subset of a member $D$ of $\mathbb{D}$ in the relative topology of $D$.

(ii) Each $\varepsilon_{t}$ is countable, and $Y$ is a free union of $Y_{t}=\cup \varepsilon_{t}, t \in T$.

Proof. By the hypotheses, we can find a point-finite or point-countable open cover $\mathcal{Q}$ of $X$ such that $\mu(U)<+\infty$ for each $U \in \mathcal{Q}$. Then for each $U \in \mathcal{Q}$, the family $\mathscr{D}_{U}=\{D \in \mathscr{D}: D \cap U \neq \varnothing\}$ is countable. Moreover, for each $D \in \mathscr{D}$, the family $\mathscr{U}_{D}=\{U \in \mathscr{Q}: D \cap U \neq \varnothing\}$ is also countable. Indeed, as each $D \in \mathscr{Q}$ satisfies the ccc, and $\mu(D \cap U)>0$ for each $U \in \mathcal{Q}_{D}$, this follows from Lemma 3.1 if $\mathscr{Q}$ is point-countable, and from $\left[\mathbf{G P}_{2}, 3.2\right]$ if $\mathscr{U}$ is point-finite. Given $U \in \mathscr{Q}$, we have $U \cap Y=U \cap \cup \mathscr{D}_{U}$, and so

$$
\begin{aligned}
\{V \cap Y: V \in \mathcal{Q}, V \cap Y \cap U \neq \varnothing\} & \subset\left\{V \cap Y: V \in \mathcal{Q}, V \cap \cup \mathscr{Q}_{U} \neq \varnothing\right\} \\
& =\left\{V \cap Y: V \in \mathcal{Q}_{D}, D \in \mathscr{Q}_{U}\right\} .
\end{aligned}
$$

Thus the family $\mathscr{Q}^{*}=\{U \cap Y: U \in \mathcal{Q}\}$ is a star-countable open (in $Y$ ) cover of $Y$. By a standard argument (see, e.g., $[\mathbf{P},(8-15)])$, there are countable families $\mathcal{U}_{t}^{*} \subset \mathcal{Q}^{*}$, $t \in T$, such that the sets $Y_{t}=\cup \mathcal{Q}_{t}^{*}$ are disjoint, and $Y=\cup_{t \in T} Y_{t}$. For $t \in T$, set

$$
\varepsilon_{t}=\left\{D \cap Y_{t}: D \in \mathscr{D}, D \cap Y_{t} \neq \varnothing\right\} \text {. }
$$

As the $Y_{t}$ 's are clopen subsets of $Y$, it suffices to show that each $\mathcal{E}_{t}$ is countable. Given $t \in T$, the point-countability of $\mathscr{U}$ implies that $\mathscr{U}_{t}=\left\{U \in \mathcal{Q}: U \cap Y \in \mathcal{Q}_{t}^{*}\right\}$ is countable, and hence so is

$$
\begin{aligned}
\mathcal{E}_{t} & =\left\{D \cap U: U \cap Y \in \mathscr{Q}_{t}^{*}, D \in \mathscr{Q}, D \cap U \neq \varnothing\right\} \\
& =\left\{D \cap U: U \in \mathscr{Q}_{t}, D \in \mathscr{Q}_{U}\right\} .
\end{aligned}
$$

3.3. Corollary. Under the assumptions of Proposition 3.2, the space $Y$ is a free union of $\sigma$-compact spaces; in particular, if $X$ is regular, then $Y$ is paracompact.

Note. In the proof of Proposition 3.2, the countability of $\mathscr{Q}_{D}$ for a point-finite $\mathscr{Q}$ could have been established by a purely topological argument (see [T, 3.8]). We used $\left[\mathbf{G P}_{\mathbf{2}}, 3.2\right]$ in order to facilitate a generalization to locally finite quasi-Radon measures (see Remark 3.5).

3.4. THEOREM. Let $\mu$ be a Radon measure in $X$ defined on a $\sigma$-algebra $\mathscr{M}$, and let $\mathscr{D}$ be a concassage of $\mu$ with $Y=\cup \mathcal{D}$. Suppose that either of the following conditions holds:

(a) $X$ is weakly $\theta$-refinable;

(b) $X$ is metalindelöf and $\mathrm{MA}+\neg \mathrm{CH}$ holds.

Then

(i) For each $\mathscr{D} * \subset \mathscr{D}, \cup \mathscr{Q} *$ is a Borel subset of $X$; in particular, $Y \in \mathscr{M}$ and $\mu(X-Y)=0$.

(ii) $\mu$ is saturated; in particular, $\mu$ is decomposable whenever it is complete.

(iii) $\mu$ is Maharam.

Proof. Suppose first that $X$ is metalindelöf and that $\mathrm{MA}+\neg \mathrm{CH}$ holds. Choose a concassage $\mathcal{E}=\cup_{t \in T} \mathcal{E}_{t}$ of $\mu$ as in Proposition 3.2, and let $Y_{t}=\cup \mathcal{E}_{t}, t \in T$. If 
$\mathcal{E}^{*} \subset \mathcal{E}$, then $E^{*}=\cup \mathcal{E}^{*}$ is a free union of the $\sigma$-compact sets $E^{*} \cap Y_{t}, t \in T$ (see 3.2(ii)). For each $t \in T$, choose an open set $V_{t} \subset X$ with $E^{*} \cap V_{t}=E^{*} \cap Y_{t}$. If $E^{*} \cap Y_{t}=\cup_{n \in \omega} K_{t, n}$ where $K_{t, n}$ are compact sets, let $L_{n}=\operatorname{cl}\left(\cup_{t \in T} K_{t, n}\right)$. Then $E^{*}=\left(\bigcup_{t \in T} V_{t}\right) \cap\left(\bigcup_{n \in \omega} L_{n}\right)$ is a Borel subset of $X$. As $\mathscr{E}$ refines $\mathscr{D}$, property (i) follows. Thus with no loss of generality, we may assume that $X=Y$.

Let $A \subset X$ be such that $A \cap B \in \Re$ for every $B \in \Re_{f}$. Then $A \cap Y_{t} \in \Re$ for each $t \in T$. Since $Y_{t}$ is $\sigma$-compact and open in $X=Y$, it is a countable union of open sets of finite measure. By $\left[\mathbf{G P}_{2}, 3.1\right]$, there are $G_{\delta}$ sets $G_{t}$ and $H_{t}$ such that

$$
A \cap Y_{t} \subset G_{t} \subset Y_{t}, \quad G_{t}-A \cap Y_{t} \subset H_{t} \subset Y_{t},
$$

and $\mu\left(H_{t}\right)=0$. As $X=Y$ is a free union of the $Y_{t}$ 's (see 3.2(ii)), the sets

$$
G=\bigcup_{t \in T} G_{t} \text { and } H=\bigcup_{t \in T} H_{t}
$$

are $G_{\delta}$. Moreover, $A \subset G, G-A \subset H$, and

$$
\mu(H)=\sum_{E \in \mathscr{E}} \mu(H \cap E)=\sum_{t \in T} \mu\left(H \cap Y_{t}\right)=\sum_{t \in T} \mu\left(H_{t}\right)=0 .
$$

By our assumptions $A \cap H$ is in $\mathfrak{M}$, and hence so is

$$
G-A=(G-A) \cap H=G \cap H-A \cap H .
$$

Consequently $A \in \Re$. In view of $\S 2,(\mathrm{~B})$, property (ii) is established.

Next suppose that $X$ is weakly $\theta$-refinable. By [P, (18.30)], there are families $\mathcal{U}_{n} \subset \mathbb{R}_{f}, n \in \omega$, of open subsets of $X$ such that for each $x \in X$, there is an $n_{x} \in \omega$ with $\left|s t\left(x, \mathscr{Q}_{n_{x}}\right)\right|=1$. Fix $n \in \omega$. The set

$$
\begin{aligned}
X_{n} & =\left\{x \in X:\left|s t\left(x, \mathscr{Q}_{n}\right)\right|=1\right\} \\
& =\left\{x \in X:\left|s t\left(x, \mathscr{Q}_{n}\right)\right| \geqslant 1\right\} \cap\left\{x \in X:\left|s t\left(x, \mathscr{Q}_{n}\right)\right| \leqslant 1\right\}
\end{aligned}
$$

is an intersection of an open and a closed set, and also a free union of $\left\{U \cap X_{n}\right.$ : $\left.U \in \mathscr{Q}_{n}\right\}$.If $\mathfrak{R}_{n}=\left\{A \in \Re^{\prime}: A \subset X_{n}\right\}$, then $\mu_{n}=\mu$ 个 $\mathfrak{R}_{n}$ is a Radon measure in $X_{n}$. For every $U \in \mathscr{Q}_{n}$, let $\mathcal{E}_{U}=\left\{D \cap U \cap X_{n}: D \in \mathscr{D}\right\}$ and $Y_{U}=\cup \mathcal{E}_{U}$. Set $\mathcal{E}=$ $\cup_{U \in \mathscr{Q}_{n}} \mathcal{E}_{U}$ and $Z=\cup \mathcal{E}$. Then $\mathscr{E}$ refines $\mathscr{D}_{n}=\left\{D \cap X_{n}: D \in \mathscr{D}\right\}$, and $Z$ is a free union of the $Y_{U}$ 's. Each element of $\mathscr{E}$ is an intersection of an open and a compact set. For every $U \in \mathscr{Q}_{n}$, the family $\mathcal{E}_{U}$ is countable, and $\mu\left(Y_{U}\right) \leqslant \mu(U)<+\infty$. Moreover, if $A \in \mathfrak{N}_{n}$, then

$$
\begin{aligned}
\mu_{n}(A) & =\sum_{D \in \mathscr{Q}} \mu_{n}(A \cap D)=\sum_{D \in \mathscr{D}} \sum_{U \in \mathscr{Q}_{n}} \mu_{n}(A \cap D \cap U) \\
& =\sum_{U \in \mathscr{Q}_{n}} \sum_{E \in \mathscr{E}_{U}} \mu_{n}(A \cap E)=\sum_{U \in \mathcal{Q}_{n}} \mu_{n}\left(A \cap Y_{U}\right) .
\end{aligned}
$$

Now by arguments analogous to those of the first part of the proof, properties (i) and (ii) are obtained for $\mu_{n}$ and $\mathscr{\mathscr { D }}_{n}$. As $X=\cup_{n \in \omega} X_{n}$, and consequently,

$$
\cup \mathscr{Q} *=\bigcup_{n \in \omega}\left[\cup\left\{D \cap X_{n}: D \in \mathscr{Q} *\right\}\right]
$$

for each $\mathscr{Q}^{*} \subset \mathscr{D}$, it is immediate that properties (i) and (ii) hold also for $\mu$ and $\mathscr{D}$. 
Finally, it is easy to see that the measure $\mu$ is Maharam if and only if its completion $\tilde{\mu}$ is. Since $\tilde{\mu}$ is a Radon measure, it is saturated and therefore decomposable by property (ii). Now property (iii) follows from $\S 2,(\mathrm{C})$.

3.5. REMARK. The quasi-Radon measures of D. H. Fremlin are complete and saturated by the definition (see $\left[\mathbf{F}_{1}, 72 \mathrm{~A}\right]$ ). If we drop these conditions from the definition, quasi-Radon measures will no longer be decomposable. However, following the arguments of $\left[\mathbf{F}_{1}, 72 \mathrm{~B}\right]$, we can still prove that each quasi-Radon measure has a "concassage" consisting of closed sets. Using this it is easy to see that for metacompact and weakly $\theta$-refinable spaces (assumption (a)), Proposition 3.2 and Theorem 3.4 remain valid for locally finite quasi-Radon measures; and using [Ok, $3.1]$ it can be seen that Corollary 3.3 is also valid, with " $\sigma$-compact" replaced by "Lindelöf". The proofs are virtually verbatim repetitions of those for Radon measures. The one-point compactification of the space $Z$ from $\left[\mathbf{F}_{2}, 2 \mathrm{~J}\right]$ shows that these results cannot be generalized to quasi-Radon measures which are not locally finite. We do not know if a generalization to metalindelöf spaces (assumption (b)) is possible, for the application of MA depends on the presence of compactness.

A space $X$ is called weakly $\delta \theta$-refinable if each open cover of $X$ has an open refinement $\cup_{n \in \omega}{ } d_{n}$ such that given $x \in X$ there is an $n_{x} \in \omega$ with $1 \leqslant\left|\operatorname{st}\left(x, \mathscr{Q}_{n_{1}}\right)\right|$ $\leqslant \omega$.

Clearly, "weakly $\delta \theta$-refinable" relates to "metalindelöf" in the same way as "weakly $\theta$-refinable" does to "metacompact". Thus it is natural to ask whether under $\mathrm{MA}+{ }_{\neg} \mathrm{CH}$, the previous results can be generalized to Radon measures in a weakly $\delta \theta$-refinable space $X$. We show that this is possible if $X$ has countable tightness.

A space $X$ has countable tightness if for each $A \subset X$ and each $x \in \operatorname{cl} A$ there is a countable set $C \subset A$ such that $x \in \mathrm{cl} C$. In particular, $X$ has countable tightness whenever it is first countable.

The next lemma is due to S. D. Dyck.

3.6. LEMma. Let $Q$ be a family of open subsets of $X$. If $X$ has countable tightness, then the set

$$
A=\{x \in X:|s t(x, Q)| \leqslant \omega\}
$$

is closed.

Proof. If $x \in \operatorname{cl} A$, choose a countable set $C \subset A$ with $x \in \mathrm{cl} C$. As

$$
s t(x, \mathbb{Q}) \subset \bigcup_{y \in C} s t(y, \mathbb{Q}),
$$

we see that $x \in A$.

3.7. Proposition. (MA $+\neg \mathrm{CH})$ Let $X$ be a weakly $\delta \theta$-refinable space of countable tightness, and let $\mu$ be a Radon measure in $X$. Then there is a concassage $\varepsilon=\cup_{n \in \omega} \varepsilon_{n}$ of $\mu$ having the following properties:

(i) For each $\mathcal{E}^{*} \subset \mathcal{E}, \cup \mathcal{E}^{*}$ is a Borel subset of $X$.

(ii) For each $n \in \omega, Y_{n}=\cup \mathcal{E}_{n}$ is a free union of $\sigma$-compact spaces.

(iii) The spaces $Y_{n}, n \in \omega$, are disjoint. 
Proof. Let $\mathfrak{N}$ be the domain of $\mu$. There are families $\mathscr{Q}_{n} \subset \mathfrak{R}_{f}, n \in \omega$, of open subsets of $X$ such that for each $x \in X$ there is an $n_{x} \in \omega$ with $1 \leqslant\left|s t\left(x, \mathscr{Q}_{n_{x}}\right)\right| \leqslant \omega$. For $n \in \omega$, let

$$
X_{n}=\left\{x \in X: 1 \leqslant\left|s t\left(x, \mathscr{Q}_{n}\right)\right| \leqslant \omega\right\}
$$

and $Z_{n}=X_{n}-\cup_{k=0}^{n-1} X_{k}$. The $Z_{n}$ 's are disjoint Borel subsets of $X$ (see Lemma 3.6), and $\cup_{n \in \omega} Z_{n}=X$. If $\Re_{n}=\left\{A \in \Re: A \subset Z_{n}\right\}$, then $\mu_{n}=\mu \uparrow \Re_{n}$ is a Radon measure in $Z_{n}$. Choose a concassage $\mathscr{D}$ of $\mu_{n}$, and set $Y_{n}=\cup \mathscr{D}$. Employing the same argument we used in the proof of Proposition 3.2, it is easy to find a concassage $\mathcal{E}_{n}=\cup_{t \in T} \mathcal{H}_{t}$ of $\mu_{n}$ such that each $\mathcal{H}_{t}$ is countable, and $Y_{n}$ is a free union of $H_{t}=\cup \mathcal{H}_{t}, t \in T$. Now it follows that $\mathscr{E}=\cup_{n \in \omega^{E}}$ is the desired concassage of $\mu$.

3.8. COROllary. (MA $\left.+{ }_{\neg} \mathrm{CH}\right)$ Let $X$ be a weakly $\delta \theta$-refinable space of countable tightness. Then each Radon measure in $X$ is saturated and Maharam.

Using Proposition 3.7, the proof of Corollary 3.8 is analogous to that of Theorem 3.4.

4. Some examples. Throughout, $R$ will denote the set of all real numbers together with its Euclidean topology.

4.1. EXAMPLE (cf. [Kr, p. 362]). Give $\omega_{1}$ the discrete topology, and set $X=\omega_{1} \times R$. If $B$ is a Borel subset of $X$, let

$$
\mu(B)=\sum_{\alpha \in \omega_{1}} \lambda\left(B^{\alpha}\right)
$$

where $B^{\alpha}=\{t \in R:(\alpha, t) \in B\}$, and $\lambda$ is Lebesgue measure in $R$. Since $X$ is a metrizable space, by 3.4(ii) and (iii), $\mu$ on $\Re$ is a saturated Radon measure, which is Maharam. Clearly, $\mu$ is not complete, and we show that it is not decomposable. Proceeding towards contradiction, suppose that $\mathscr{H}$ is a decomposition of $\mu$. It is easy to find a discrete family $\left\{H_{\alpha}: \alpha \in \omega_{1}\right\} \subset \mathcal{H}$ such that each $H_{\alpha}$ contains an uncountable compact set $K_{\alpha}$. Using [Kr, §30, XIV and $\S 36$, V, Corollary 2], we see that each $H_{\alpha}$ contains a Borel set $B_{\alpha}$ which is not of Borel class $\alpha$ (see [ $\left.\mathbf{K r}, \S 30, \mathrm{II}\right]$ ). Then $B=\cup_{\alpha \in \omega_{1}} B_{\alpha}$ is not a Borel set, while $B \cap H \in \mathscr{B}$ for each $H \in \mathscr{H}$; a contradiction.

4.2. EXAMPLE. This example is similar to that in [GrP, Example 7]. Detailed proofs of properties we quote are given in $\left[\mathbf{G P}_{\mathbf{3}}, 12.7\right]$.

For $k, n \in \omega$, let $q_{k, n}=\left(k 2^{-n}, 2^{-n}\right)$ and $Q=\left\{q_{k, n}: k=0, \ldots, 2^{n} ; n \in \omega\right\}$. In $X=[0,1] \cup Q$, we define a topology as follows: the points of $Q$ are isolated, and a neighborhood base at $t \in[0,1]$ is given by the sets

$$
U(t, \varepsilon)=\{t\} \cup\left\{q_{k, n} \in Q: 2\left|k 2^{-n}-t\right|<2^{-n}<\varepsilon\right\}
$$

where $\varepsilon>0$. Thus $U(t, \varepsilon)$ consists of $t$ and of all points from $Q$ which lie inside the open wedge in $[0,1]^{2}$ with vertex $(t, 0)$ height $\varepsilon$, and the slopes of the sides equal to \pm 2 .

With this topology, $X$ is a zero-dimensional, $\theta$-refinable, locally compact space. Each subset of $X$ is Borel. As $X$ is separable but not Lindelöf, it is not metalindelöf. 
Let $g\left(q_{k, n}\right)=2^{-n}$ for $n \in \omega$ and $k=0, \ldots, 2^{n}$, and $g(t)=1$ for $t \in[0,1]$. If $B \subset X$, set

$$
\mu(B)=\sum_{x \in B} g(x) .
$$

Then $\mu$ is a Radon measure in $X$, and $\mathscr{D})=\{\{x\}: x \in X\}$ is a concassage of $\mu$. Thus $\cup \mathscr{Q}=X$ is not paracompact.

For further examples we need a result of K. Kunen (see $[\mathbf{K n}])$.

4.3. Proposition. (CH) There is a zero-dimensional, nonseparable, compact space $Y$ which is hereditarily Lindelöf. Moreover, there is a Radon probability $\nu$ defined on Borel subsets of $Y$ such that $\nu(B)=0$ if and only if $B$ is a nowhere dense Borel subset of $Y$.

4.4. EXAmple. $(\mathrm{CH})$ The space $X$ of Example 4.2 is not metalindelöf because it is separable. We destroy its separability by replacing the points of $Q$ with copies of the space $Y$ from Proposition 4.3. We adhere to the notation of Example 4.2. Detailed proofs of properties we state are given in $\left[\mathbf{G P}_{\mathbf{2}}, 3.7\right.$ or $\left.\mathbf{G P}_{\mathbf{3}}, 12.12\right]$.

Let $Z=[0,1] \cup(Q \times Y)$. Using $\mathrm{CH}$, we can find a point-countable family $\left\{G_{t}\right.$ : $t \in[0,1]\}$ of clopen subsets of $Y$. If $z=(q, y)$ is in $Q \times Y$, we let the neighborhood base of $z$ in $Z$ be the same as that of $z$ in $\{q\} \times Y$. The neighborhood base at $t \in[0,1]$ is given by the sets

$$
\{t\} \cup\left([U(t, \varepsilon)-\{t\}] \times G_{t}\right)
$$

where $\varepsilon>0$.

With this topology, $Z$ is a zero-dimensional, $\theta$-refinable, locally compact, and metalindelöf space. By $\left[\mathbf{G P}_{\mathbf{2}}, 3.2\right]$, no uncountable subfamily of $\left\{G_{t}: t \in[0,1]\right\}$ is point-finite. From this and the fact that the space $X$ from Example 4.2 is not metalindelöf, it is easy to see that $Z$ is not metacompact.

For a Borel set $B \subset Z$, let

$$
\mu(B)=\sum_{q \in Q} g(q) \cdot \nu[B \cap(\{q\} \times Y)]+\sum_{t \in B \cap[0,1]} g(t) .
$$

Then $\mu$ is a Radon measure in $X$, and

$$
\mathscr{D}=\{\{q\} \times Y: q \in Q\} \cup\{\{t\}: t \in[0,1]\}
$$

is a concassage of $\mu$. Thus $\cup \mathscr{Q}=Z$ is not paracompact.

4.5. EXAMPLE. $(\mathrm{CH})$ Our aim is to obtain a metalindelöf space $Z$ and a Radon measure $\mu$ in $Z$ which is not Maharam.

We begin with a construction analogous to that in $\left[\mathbf{G P}_{\mathbf{1}}, \S 4\right]$. However, rather than using the idea of A. J. Ostaszewski (which requires $\diamond-$ see [Os, §2]), we shall elaborate on that of I. Juhász, K. Kunen and M. E. Rudin (see [JKR, §1]).

Let $I=2^{\omega}$ have the product topology, and let $\lambda$ be the unique Haar probability in I. If

$$
U(t, n)=\{u \in I: u \uparrow n=t \uparrow n\}
$$

where $t \in I$ and $n \in \omega$, then $\lambda[U(t, n)]=2^{-n}$. Using $\mathrm{CH}$, fix an enumeration $\left\{t_{\alpha}\right.$ : $\left.\alpha \in \omega_{1}\right\}$ of $I$ such that $\left\{t_{n}: n \in \omega\right\}$ is dense in $I$, and let $I_{\alpha}=\left\{t_{\beta}: \beta \in \alpha\right\}$ for each 
$\alpha \in \omega_{1}$. Set $X=I^{2}$, and define $\pi: X \rightarrow I$ by $\pi\left(t_{\alpha}, t\right)=t_{\alpha}$ for each $\left(t_{\alpha}, t\right) \in X$. If $E \subset X$ and $\alpha \in \omega_{1}$, we let

$$
E^{\alpha}=\left\{t \in I:\left(t_{\alpha}, t\right) \in E\right\} .
$$

By $\mathrm{CH}$, there is an enumeration $\left\{S_{\alpha}: \alpha \in \omega_{1}\right\}$ of all sets $S \subset X$ such that $|\pi(S)|=\omega$ and $\left|S^{\gamma}\right|=1$ for each $\gamma \in \pi(S)$. We may assume that $\pi\left(S_{0}\right)=I_{\omega}$. For each $\alpha \in \omega_{1}-\omega$, the family

$$
\delta_{\alpha}=\left\{S_{\beta}: \beta \in \alpha, S_{\beta} \subset I_{\alpha} \times I, t_{\alpha} \in \operatorname{cl}\left[\pi\left(S_{\beta}\right)\right]\right\}
$$

is countable and nonempty; for $S_{0} \in \mathcal{S}_{\alpha}$.

Now proceeding as in $\left[\mathbf{G P}_{3}, 13.14\right]$, we topologize $X$ so that the following conditions are satisfied:

(i) $X$ is a zero-dimensional, first countable, and locally compact space;

(ii) for each $\alpha \in \omega_{1}, I_{\alpha} \times I$ is an open subset of $X$, and the subspace $\left\{t_{\alpha}\right\} \times I$ has the product topology;

(iii) if $\omega \leqslant \alpha<\omega_{1}$ and $S \in \mathcal{S}_{\alpha}$, then $\left\{t_{\alpha}\right\} \times I \subset \operatorname{cl} S$;

(iv) each $x \in X$ has a neighborhood $U$ with $\Sigma_{\alpha \in \omega_{1}} \lambda\left(U^{\alpha}\right)<+\infty$.

The detailed proofs of these assertions are given in $\left[\mathbf{G P}_{3}, 13.14\right]$.

Claim 1. If $B$ is a Borel subset of $X$, then $B^{\alpha}, \alpha \in \omega_{1}$, is a Borel subset of $I$, and

$$
\left|\left\{t_{\alpha} \in \pi(B): B^{\alpha} \neq I\right\}\right| \leqslant \omega .
$$

Proof. The first part of the claim follows immediately from (ii). Let $F \subset X$ be closed. Since $\pi(F)$ is a separable subset of $I$, there is an $S_{\beta} \subset F, \beta \in \omega_{1}$, with $\operatorname{cl}\left[\pi\left(S_{\beta}\right)\right]=\operatorname{cl}[\pi(F)]$. Find an infinite $\kappa \in \omega_{\mathrm{i}}$ with $S_{\beta} \subset I_{\kappa} \times I$. If $\alpha \in \omega_{1}, \alpha>\kappa$, and $t_{\alpha} \in \mathrm{cl}\left[\pi\left(S_{\beta}\right)\right]$, then $S_{\beta} \in \mathcal{\delta}_{\alpha}$. Thus by (iii),

$$
\left\{t_{\alpha}\right\} \times I \subset \mathrm{cl} S_{\beta} \subset F
$$

for each $t_{\alpha} \in \pi(F)$ with $\alpha>\kappa$. It follows that $F$ belongs to the family $\mathbb{Q}$ of all sets $A \subset X$ for which

$$
\left|\left\{t_{\alpha} \in \pi(A): A^{\alpha} \neq I\right\}\right| \leqslant \omega .
$$

As $Q$ is a $\sigma$-algebra in $X$, the proof is completed.

Let $Y$ and $\nu$ be as in Proposition 4.3. Since $I$ is second countable, $\eta=\lambda \times \nu$ is a Radon probability in $I \times Y$. If $B$ is a Borel subset of $X \times Y, \alpha \in \omega_{1}$, and $t \in I$, then

$$
\begin{aligned}
B^{\alpha} & =\left\{(t, y) \in I \times Y:\left(t_{\alpha}, t, y\right) \in B\right\}, \\
B^{\alpha, t} & =\left\{y \in Y:\left(t_{\alpha}, t, y\right) \in B\right\},
\end{aligned}
$$

are Borel subsets of $I \times Y$ and $Y$, respectively. Note that $(E \times Y)^{\alpha}=E^{\alpha} \times Y$ for each Borel set $E \subset X$. We let

$$
\mu(B)=\sum_{\alpha \in \omega_{1}} \eta\left(B^{\alpha}\right)
$$

for each Borel $B \subset X \times Y$. Using (iv), it is easy to check that $\mu$ is a Radon measure in $X \times Y$.

By $\mathrm{CH}$, there is an enumeration $\left\{y_{\alpha}: \alpha \in \omega_{1}\right\}$ of $Y$. If $G_{\alpha}=Y-\operatorname{cl}\left\{y_{\beta}: \beta \in \alpha\right\}$, then $\mu\left(G_{\alpha}\right)=1$; for by Proposition 4.3 countable subsets of $Y$ are nowhere dense. 
Since the $G_{\alpha}$ 's are nested and $\bigcap_{\alpha \in \omega_{1}} G_{\alpha}=\varnothing$, the family $\left\{G_{\alpha}: \alpha \in \omega_{1}\right\}$ is point-count able.

Claim 2. In the relative topology, the space

$$
Z=\bigcup_{\alpha \in \omega_{1}}\left(I_{\alpha+1} \times I \times G_{\alpha}\right)
$$

is locally compact and metalindelöf.

Proof. As by (ii), $Z$ is an open subset of $X \times Y$, it is locally compact. Given an open cover $\mathcal{Q}$ of $Z$, find a common open refinement $\mathcal{T}$ of $\mathcal{Q}$ and $\left\{I_{\alpha+1} \times I \times G_{\alpha}\right.$ : $\left.\alpha \in \omega_{1}\right\}$. Since both $I_{\alpha+1} \times I$ and $G_{\alpha}$ are $\sigma$-compact, so is $I_{\alpha+1} \times I \times G$. Thus $T$ has a subcover $\check{\tau}_{*}$ such that

$$
\left|\left\{V \in \Upsilon_{*}: V \subset I_{\alpha+1} \times I \times G\right\}\right| \leqslant \omega
$$

for each $\alpha \in \omega_{1}$. Now the point-countability of $\left\{G_{\alpha}: \alpha \in \omega_{1}\right\}$ implies that of $\widetilde{\Upsilon}_{*}$.

The restriction of $\mu$ to the Borel subsets of $Z$, denoted also by $\mu$, is a Radon measure in $Z$. We show that it is not Maharam.

If $B$ is a Borel subset of $Z$, denote by $B^{*}$ the class determined by $B$ in the measure algebra of $\mu$. For $k=0,1$ and $\alpha \in \omega_{1}$, let

$$
A_{k}=\{t \in I: t(0)=k\} \text { and } E_{k, \alpha}=\left\{t_{\alpha}\right\} \times A_{k} \times G_{\alpha} .
$$

Then $E_{k, \alpha}$ are closed subsets of $Z$, and we assume that

$$
\sup \left\{E_{0, \alpha}^{\cdot}: \alpha \in \omega_{1}\right\}=E^{\cdot}
$$

for some Borel set $E \subset Z$. Then given $\alpha \in \omega_{1}$, we have

$$
E_{0 . \alpha}^{\cdot} \leqslant E^{\cdot} \leqslant\left(Z-E_{1, \alpha}\right)^{\cdot} \text {, }
$$

and so

$$
\begin{gathered}
\int_{A_{0}} \nu\left(G_{\alpha}-E^{\alpha, t}\right) d \lambda(t)=\eta\left(A_{0} \times G_{\alpha}-E^{\alpha}\right)=\mu\left(E_{0, \alpha}-E\right)=0, \\
\int_{A_{1}} \nu\left(E^{\alpha, t}\right) d \lambda(t)=\eta\left[E^{\alpha} \cap\left(A_{1} \times G_{\alpha}\right)\right] \\
=\mu\left(E \cap E_{1, \alpha}\right)=\mu\left[E-\left(Z-E_{1, \alpha}\right)\right]=0 .
\end{gathered}
$$

Now it follows from Claim 1 that $\left\{\left(t_{\alpha}, t\right) \in X: \nu\left(E^{\alpha, t}\right)>\frac{1}{2}\right\}$ is not a Borel subset of $X$. As $X$ is first countable, this is a contradiction; for by a result of R. A. Johnson, the function $\left(t_{\alpha}, t\right) \mapsto \nu\left(E^{\alpha, t}\right)$ is Borel measurable (see $[\mathbf{J}, 1.1]$ or $\left.\left[\mathbf{G P}_{3}, 7.10\right]\right)$.

We showed that in the space $Z$ from the previous example, properties (ii) and (iii) of Theorem 3.4 are false (see $\$ 2,(B)$ and (C)). Next we show that property (i) of Theorem 3.4 is also false in $Z$.

4.6. EXAmple. $(\mathrm{CH})$ We shall use the notation of Example 4.5. For each $\alpha \in \omega_{1}$, choose an open compact set $K_{\alpha} \subset G_{\alpha}$ with $\nu\left(K_{\alpha}\right)>\frac{1}{2}$, and if $B \subset Z$ is a Borel set, let

$$
\mu_{1}(B)=\sum_{\alpha \in \omega_{1}} \eta\left[B^{\alpha} \cap\left(A_{0} \times K_{\alpha}\right)\right] .
$$

Then $\mu_{1}$ is a Radon measure in $Z$, and

$$
\mathscr{D}=\left\{\left\{t_{\alpha}\right\} \times A_{0} \times K_{\alpha}: \alpha \in \omega_{1}\right\}
$$


is a concassage of $\mu_{1}$. Now arguing as in Example 4.5, it is easy to see that $\cup \mathscr{D}$ is not a Borel subset of $Z$.

Note. In $\left[\mathbf{F}_{2}, 2 \mathrm{C}\right]$, without any set theoretic axioms, D. H. Fremlin constructed a completely regular space $X$ and a Radon measure $\mu$ in $X$ which does not satisfy properties (ii) and (iii) of Theorem 3.4. Naturally, the space $X$ cannot be metalindelöf. An easy modification of the measure also provides a counterexample to property (i) of Theorem 3.4 .

\section{REFERENCES}

[F $\left.\mathbf{F}_{1}\right]$ D. H. Fremlin, Topological Riesz spaces and measure theory, Cambridge Univ. Press, London, 1974.

$\left[\mathbf{F}_{2}\right] \ldots$, Topological measure spaces: two counter-examples, Math. Proc. Cambridge Philos. Soc. 78 $(1975), 95-106$.

$\left[\mathbf{F}_{\mathbf{3}}\right] \ldots$ Decomposable measure spaces, Z. Wahrsch. Verw. Gebiete 45 (1978), 159-167.

$\left[\mathbf{G P}_{1}\right]$ R. J. Gardner and W. F. Pfeffer, Are diffused, regular, Radon measures $\sigma$-finite?, J. London Math. Soc. (2) 20 (1979), 485-494.

$\left[\mathbf{G P}_{2}\right] \ldots$ Some undecidability results concerning Radon measures, Trans. Amer. Math. Soc. 259 $(1980), 65-74$.

$\left[\mathbf{G P}_{3}\right] \ldots$ B Borel measures, Handbook of Set-Theoretic Topology, North-Holland, Amsterdam (to appear).

[GrP] G. Gruenhage and W. F. Pfeffer, When inner regularity of Borel measures implies regularity, J. London Math. Soc. (2) 17 (1978), 165-171.

[II] A. Ionescu Tulcea and C. Ionescu Tulcea, Topics in the theory of lifting, Springer-Verlag, Berlin, Heidelberg and New York, 1969.

[J] R. A. Johnson, Products of two Borel measures, Trans. Amer. Math. Soc. 269 (1982), 611-625.

[JKR] I. Juhász, K. Kunen and M. E. Rudin, Two more hereditarily separable non-Lindelöf spaces, Canad. J. Math. 28 (1976), 998-1005.

[Kn] K. Kunen, A compact L-space, Topology Appl. 12 (1981), 283-287.

[Kr] K. Kuratowski, Topology, Vol. 1, Academic Press, New York, 1966.

[Ok] S. Okada, Supports of Borel measures, J. Austral. Math. Soc. Ser. A 27 (1979), 221-231.

[Os] A. J. Ostaszewski, On countably compact, perfectly normal spaces, J. London Math. Soc. (2) 14 (1976), 505-516.

[P] W. F. Pfeffer, Integrals and measures, Marcel Dekker, New York, 1977.

[T] F. D. Tall, The countable chain condition versus separability-applications of Martin's axiom, Gen. Topology Appl. 4 (1974), 315-339.

Department of Mathematics, University of Petroleum and Minerals, Dhahran, Saudi Arabia Department of Mathematics, University of California, Davis, California 95616 